\title{
Adaptive Optics for the CHARA Array
}

Theo A. ten Brummelaar ${ }^{\mathrm{a}}$, Laszlo Sturmann ${ }^{\mathrm{a}}$, Judit Sturmann ${ }^{\mathrm{a}}$, Stephen T. Ridgway ${ }^{\mathrm{b}}$, John D. Monnier $^{c}$, Michael J. Ireland ${ }^{\mathrm{d}, \mathrm{f}}$, Xiao Che ${ }^{\mathrm{c}}$, Harold A. McAlister ${ }^{\mathrm{e}}$, Nils H. Turner ${ }^{\mathrm{a}}$, \& P.G. Tuthill $^{\mathrm{f}}$ ${ }^{a}$ The CHARA Array of Georgia State University, Mount Wilson Observatory, Mount Wilson CA USA 91023; ' National Optical Astronomy Observatory, 950 North Cherry Ave., Tucson AZ USA 85719, ${ }^{\mathrm{C}}$ University of Michigan, 500 Church Street, Ann Arbor, MI USA 48109-1090, ${ }^{\mathrm{d}}$ Macquarie University, Balaclava Road, North Ryde, NSW Australia 2109, ${ }^{\mathrm{e}} \mathrm{CHARA}$ Georgia State University, 1 Park Place, Atlanta, GA USA 30303-3696, ${ }^{\mathrm{f}}$ The Unviersity of Sydney, NSW 2006, Australia. ${ }^{\mathrm{f}}$ Australian Astronomical Observatory, PO Box 296, Epping, NSW 2121, Australia

\begin{abstract}
The CHARA Array is a six telescope optical/IR interferometer run by the Center for High Angular Resolution Astronomy of Georgia State University and is located at Mount Wilson Observatory just to the north of Los Angeles California. The CHARA Array has the largest operational baselines in the world and has been in regular use for scientific observations since 2004. In 2011 we received funding from the NSF to begin work on Adaptive Optics for our six telescopes. Phase I of this project, fully funded by the NSF grant, consists of designing and building wavefront sensors for each telescope that will also serve as tip/tilt detectors. Having tip/tilt at the telescopes, instead of in the laboratory, will add several magnitudes of sensitivity to this system. Phase I also includes a slow wavefront sensor in the laboratory to measure non-common path errors and small deformable mirrors in the laboratory to remove static and slowly changing aberrations. Phase II of the project will allow us to place high-speed deformable mirrors at the telescopes thereby enabling full closed loop operation. We are currently seeking funding for Phase II. This paper will describe the scientific rational and design of the system and give the current status of the project.
\end{abstract}

Keywords: Interferometry, Tip/Tilt, Adaptive Optics

\section{INTRODUCTION}

In 2011 the NSF funded Phase I of the Center for High Angular Resolution Astronomy (CHARA ${ }^{1}$ ) Array Adaptive Optics (AO) program. Phase I includes the installation of a wavefront sensor (WFS) including dichroic splits, input optics and alignment optics, at each of our six 1m telescopes; a slower WFS in the laboratory to measure non-commonpath errors; and small low order deformable mirrors in the laboratory to remove static and slowly changing aberrations in the optical system. Phase II, as yet unfunded, will include the addition of fast high-order deformable mirrors and a full closed loop AO system. The wavefront sensors will provide tip/tilt measurement in order to close the tip/tilt loop locally at the telescopes, rather than using the existing tip/tilt sensor in the laboratory, which will be modified and become the slower WFS in the laboratory to detect non-common path errors. Having tip/tilt sensing at the telescopes, rather than in the laboratory, will improve the tip/tilt magnitude limit by several magnitudes in the V band used for tip/tilt.

The project is still in its initial stages and we are currently purchasing the major optical elements and detector systems. The optic design is nearly complete. In this paper we will briefly describe the scientific rational for the addition of AO to the CHARA Array, as well as the current status of the optical design.

\section{TECHNICAL JUSTIFICATION}

The addition of AO to CHARA rests on the proven technical foundation of the CHARA facility. CHARA has demonstrated the faintest limiting magnitudes in the visible $(\mathrm{R}=9)$ of any interferometer. In the infrared, the current CHARA K=8.5 faint star limit compares favorably with the Keck and VLTI interferometers, especially considering these systems have $>70 \mathrm{x}$ more collecting area. Nevertheless, obtaining a fainter limiting magnitude will open many new areas of science for CHARA, and the addition of AO to the Array will do this. Furthermore, AO will greatly enhance the scientific productivity of the Array by significantly increasing the number of nights on which good data can be collected. Like all interferometers, the CHARA Array's data quality is a very strong function of the quality of seeing. By 
improving the wavefront quality many more nights will be suitable for imaging the fainter targets. In short, the addition of AO to the Array will provide the following three significant areas of technical improvement.

\subsection{Eliminating 12 optical surfaces radically boosts visible tip/tilt tracking}

Currently, a common camera for all telescopes located in the beam combiner laboratory ${ }^{2}$ does the tip/tilt sensing. A fundamental advantage of the AO upgrade hinges upon moving the wavefront tip/tilt detection upstream from the lab into the individual telescope domes. By detecting the photons necessary for tip/tilt tracking at the telescope, we eliminate an extra 19 optical surfaces and associated aberrations, while adding only 7 in the feed optics to the WFS, and thereby increase the tip/tilt limiting magnitude by a seeing dependent factor of $\sim 10-30$. An additional boost is achieved by a state-of-the-art low-noise electron-multiplied CCD camera at each telescope. The increase in throughput also enables the inclusion of a low-order AO system while maintaining the dramatic improvement in tip/tilt tracking limiting magnitude. The improved R-band tracking is critical to increasing the number of observable young stellar objects and other dusty objects.

\subsection{Higher fringe coherence directly improves the infrared limiting magnitude}

In interferometry the most important quantity is the "system visibility" which quantifies the degree of coherence between two interferometer beams and this system visibility is closely linked to the Strehl ratio of the optical system ${ }^{3}$. Our simulations show adaptive optics will provide system visibility gains in the near-infrared equivalent to 0.9 (K band) to 2.9 (J band) magnitudes. Indeed, the large J-band improvement will open up a new waveband that has not been utilized at all at CHARA to date. AO wavefront stabilization will give more positive control over focus, alignment, and the pointing offsets required to compensate for atmospheric refraction. Table 1 shows that substantial improvement here will be achieved even with Phase I only because this stage includes a lab deformable mirror to correct for static aberrations in the optical train including the primary/secondary system. The full AO upgrade will have huge advantages at $\mathrm{J}$ band with diminishing returns beyond $\mathrm{K}$ band since $\mathrm{r}_{0}$ becomes comparable to the $1 \mathrm{~m}$ apertures of the CHARA telescopes.

Higher Strehl ratios are worth more than just better limiting magnitudes. For imaging studies of well-resolved objects in the photon-limit, signal-to-noise goes as the square of the visibility, which is effectively the square of the Strehl. Furthermore, better infrared performance can also be leveraged for greater visible light sensitivity since CHARA routinely uses its IR combiners as fringe trackers for the visible PAVO and VEGA combiners.

Table 1. Sensitivity Improvements (magnitudes) using Adaptive Optics at CHARA (value in parenthesis shows Phase I only "Static Correction + Tip/Tilt" upgrade

$\begin{array}{ccccc}\text { Seeing } & \begin{array}{c}\text { R band } \\ \text { (tip/tilt tracking) }\end{array} & \text { J band } & \text { H band } & \text { K band } \\ \text { "Poor" seeing }\left(\mathrm{r}_{0}=7.0 \mathrm{~cm}\right) & +2.7(+2.7) & +3.5(+2.1) & +1.8(+1.1) & +1.1(+0.7) \\ \text { "Excellent" seeing }\left(\mathrm{r}_{0}=12.0 \mathrm{~cm}\right) & +4.7(+4.7) & +2.9(+1.9) & +1.5(+1.0) & +0.9(+0.6)\end{array}$

\subsection{Wavefront stability eliminates drop-outs in fringe tracking and single mode fibers}

The AO system will stabilize the wavefront quality so that the system visibility is not just higher on average but is significantly more stable, especially at shorter wavelengths. The increased stability is important for continuous tracking of atmospheric turbulence for the infrared fringe tracking of faint sources, since even a short (few coherence time) drop in system visibility could mean total loss of fringe lock and increased overhead to re-acquire fringes. Indeed, fringe dropouts represent a basic limit of present systems even beyond photon or read noise (see detailed modeling of Lawson et $a l^{4}{ }^{4}$. More accurate visibility measurements and measurements of smaller visibilities both contribute to substantial gains in dynamic range, with improved detection of faint companions (e.g. Exoplanets) and low surface brightness image structure.

\section{SCIENTIFIC JUSTIFICATION}

These three enhancements will enrich the reach of existing science projects with an order-of-magnitude more targets, improved precision, higher dynamic range, better image quality, and enabling in some cases higher spectral and/or spatial resolution. They will also improve the fraction of usable nights, resulting in more science productivity. The 
performance gains will also open previously unobtainable fields of astronomy. We discuss here specifically a breakthrough survey of T Tauri disks that will be possible with AO on CHARA and will highlight other significant new discovery areas.

\subsection{Key Project: Revealing the nature of T Tauri Stars}

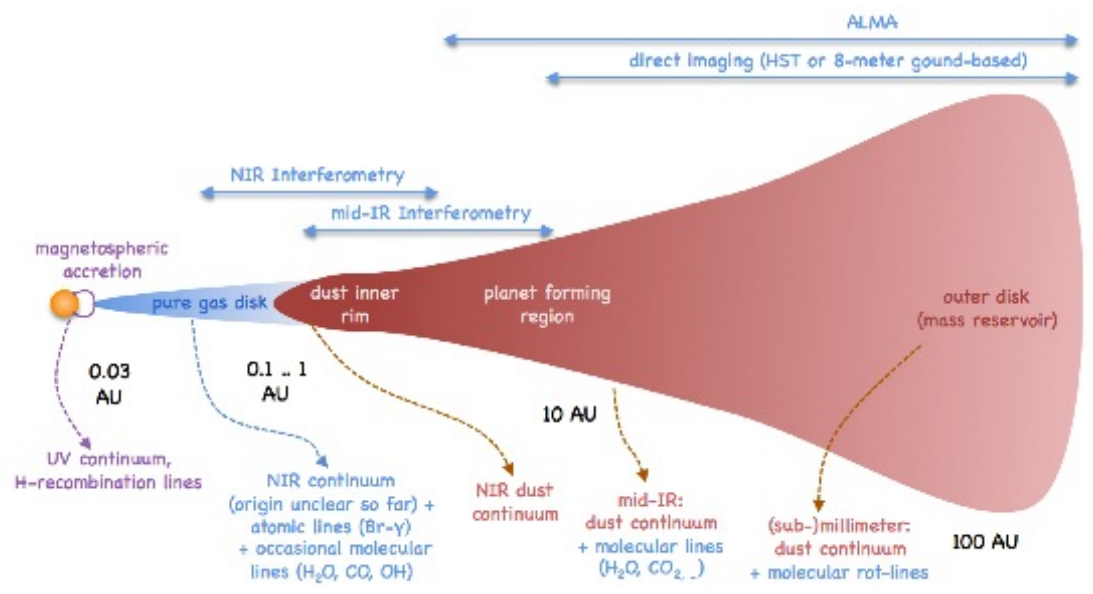

Figure 1. Schematic of a YSO disk (from Dullemond \& Monnier ${ }^{5}$ )

Infrared interferometers have made fundamental advances in our models of the inner AU of circumstellar disks around young stars. Millan-Gabet et $\mathrm{al}^{6,7}$ first discovered that the dust evaporation radii at the inner edge of intermediate-mass Herbig Ae stars were many times larger than expected from the conventional disk models at the time. The first generation of interferometry results were summarized in Monnier \& Millan-Gabet ${ }^{8}$, introducing the now wellestablished YSO size-luminosity relation. The large disk sizes were elegantly explained by the work of Natta et $a l^{9}$ and Dullemond, Dominik \& Natta ${ }^{10}$, using the mechanism of a "puffed-up inner wall" of dust directly heated by stellar radiation at the inside edge of the dust-rich disk. While these insights were first established for the higher mass and brighter Herbig Ae stars, they have had widespread implications for other young disks, especially the young solar analogues - the T Tauri stars. Figure 1 shows a schematic of a proto-planetary disk with the relevant regions labeled here we are most sensitive to the near-IR emitting region within $1 \mathrm{AU}$ of the central young star.

Akeson $e t a l^{11}$ and Eisner et $a l^{12}$ showed that T Tauri stars also have a size luminosity relation, although the sizes appear to be even larger than expected from the analogous Herbig Ae relationship (see Figure 2a). Various mechanisms have been suggested to explain these results, including scattering ${ }^{13}$ accretion heating, and reprocessing of the energy released as matter falls on the $\operatorname{star}^{14}$. Answers to this puzzle have been difficult to determine since $\mathrm{T}$ Tauri disks are much smaller in physical size than for the more luminous Herbig objects and also the disk emission is relatively less strong in the infrared. To make progress, we must increase our angular resolution and collect multi-wavelength data to constrain the size scale and emission mechanisms. Ideally, we would like real-time imaging to see how the disk structure varies with time and might respond to the fluctuating luminosity of the central source from variable accretion flows.

With this AO upgrade, young star studies at CHARA will be revolutionized. To date, there have been only three published YSO results from CHARA. We are currently collecting data on 14 new targets - a time consuming process typically using the most sensitive single baseline beam combiner.

In order to illustrate how the combined $\mathrm{R}$ band and $\mathrm{K}$ band improvements affect the number of YSOs observable by CHARA, we have plotted all the Taurus YSOs (nearly all are T Tauri stars) from Kenyon \& Hartmann ${ }^{15}$ in Figure 2 b. There are approximately 11 YSOs (in Taurus) within the current reach of CHARA, while the number of targets increases to 61 when we consider the performance improvements using the AO system for median seeing. Specifically, we gain 25 targets with a tip/tilt only upgrade that increases R-band sensitivity, an additional 25 sources by including a static wavefront corrector to fix beam-train static aberrations and using the full AO system. This simple estimate does not take into account that YSOs are generally resolved, which would make the full AO correction more critical. Also, as stated above, the AO advantage comes more dramatically at shorter wavelengths ( $\mathrm{J}$ and $\mathrm{H}$ band) while this figure focuses just 
on $\mathrm{K}$ band. These upgrades will provide the crucial sensitivity boost required to keep CHARA leading in YSO interferometry for the coming decade.
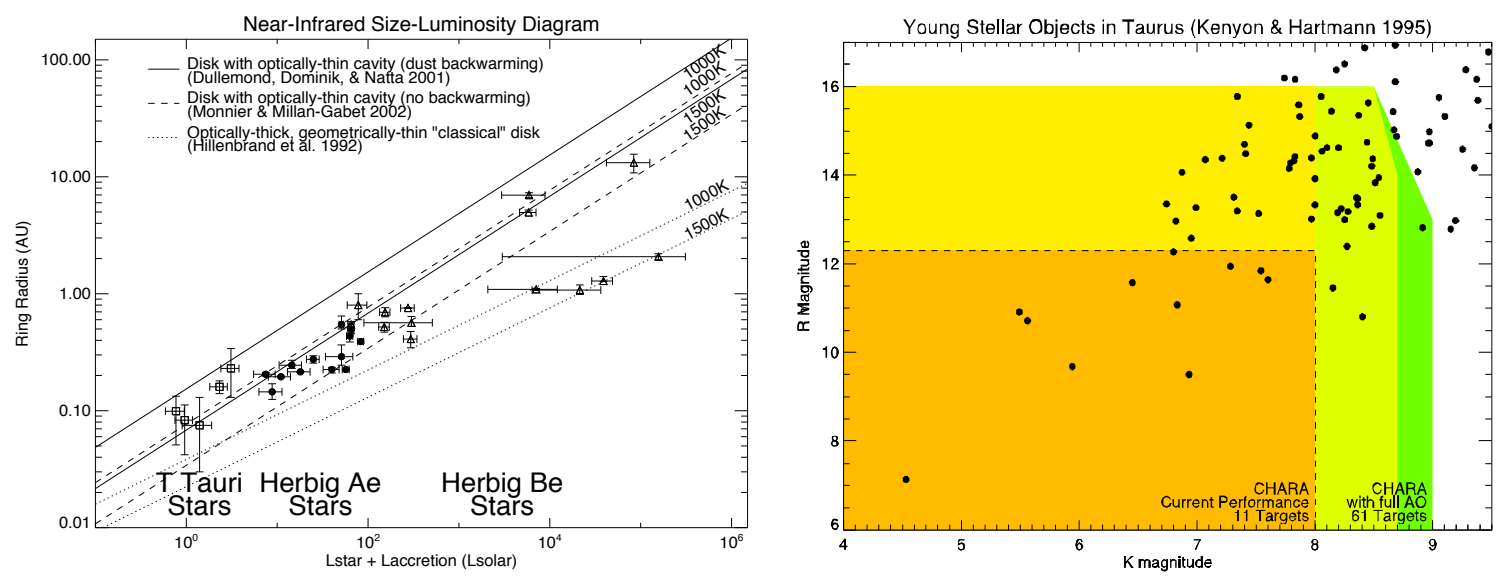

Figure 2. (A Left). The well-known (near-infrared) size-luminosity diagram for YSO disks (reprinted here from Dullemond \& Monnier ${ }^{4}$ ). T Tauri disks are observed to be unexpectedly "over-sized" compared to their higher mass Herbig Ae counterparts. (B Right) The combined improvement in $\mathrm{R}$ band and $\mathrm{K}$ band limiting magnitudes will have a transformative effect on the study of Young Stars with CHARA (figure reflects improvement for median seeing). CHARA with AO can observe approximately five times more YSOs than the present system - virtually all the new sources are $\mathrm{T}$ Tauri stars. The other shaded regions show the incremental gains for tip/tilt only, tip/tilt with static wavefront correction, and full AO.

\subsection{New discovery areas: bringing breakthrough sensitivity to CHARA}

Up to now, the only interferometers able to measure fringes on objects fainter than $\mathrm{K}>8$ have required 8 - $\mathrm{m}$ class telescopes (KI, VLTI). The priority to increase CHARA sensitivity is responsive to the demands of the community: many of the most competitive proposals from the recent CHARA public time (administered through NOAO) focused on the faintest targets. Our AO upgrades will open up discovery areas for studying new classes of objects, including Active Galactic Nuclei (AGN) and Microquasars. Here, the full AO corrections are needed to take full advantage of the 1-m collecting area to make these faint objects feasible.

The brightest AGN (NGC 4151, NGC 1068, Whirlpool, etc.) have a coherent flux of approximately K=9 (from the central engine only, not including the entire galaxy), and have been tracked at KI and VLTI ${ }^{16,17}$. Our AO models predict that a few of the brightest AGN will be observable under the best seeing conditions at CHARA - this would truly be a breakthrough capability since CHARA possesses 3-5 times better resolution than other facilities and AGN have only been partially resolved to date in the near-IR. Recent conceptual advances using clumpy dusty tori ${ }^{18}$ in AGN have rested on the new generation of mid-infrared VLTI measurements and predict clear spatial signatures with CHARA angular resolution of $<1$ mas.

Microquasars represent an exotic class of objects that can be studied with an upgraded CHARA under excellent seeing conditions. The term Microquasars is given to a somewhat disparate population of systems in which a comparatively normal star is in orbit around a compact object such as a white dwarf, neutron star or black hole. Due to accretion onto the compact component, microquasars present astronomy with a window on truly unique phenomena. They are relatively near-field analogs of AGN and are laboratories for a wealth of unique physics including jets and accretion disks. SS433 is one of the brightest examples $(\mathrm{R}=12.2, \mathrm{~K}=8.2)$ and should be observable with the these upgrades. Because the underlying engine entails highly relativistic physics, studies have the potential for fundamental discovery with farreaching impact. More specifically, interferometric measurements could contribute significantly to (1) characterizing the central dusty disk (2) understanding the connection between the accretion disk and the radio jet and (3) placing observational constraints on specific accretion/jet formation models. Other systems involving blackhole/NS star components will also be observable, including Cyg X-1, Vela X-1, LS5039, and LS I+61-303. 


\section{OPTICAL DESIGN}

When compared to standard single telescope imaging, the telescopes of an interferometer are very small field of view on-axis systems, and from that point of view the requirements placed on an AO system for these telescopes are not particularly difficult to meet. Furthermore, at CHARA the telescope diameter is $1 \mathrm{~m}$ and so we do not require a large number of actuators. Two decades of AO development for astronomy have produced a rich technology and body of experience and the problem that we wish to solve requires a milli-arcsecond field of view, on-axis system using of order 30 sub-pupils. Such systems are not necessarily complex and expensive ${ }^{19}$, allowing us to use a more pragmatic approach than the intensive development needed for large telescopes. Nevertheless, an interferometric array does offer a number of special features. The CHARA telescopes (Figure 3) produce a $125 \mathrm{~mm}$ afocal, tilt-corrected beam. CHARA also has optical paths of hundreds of meters and many optical surfaces between the telescope and the beam combiner.

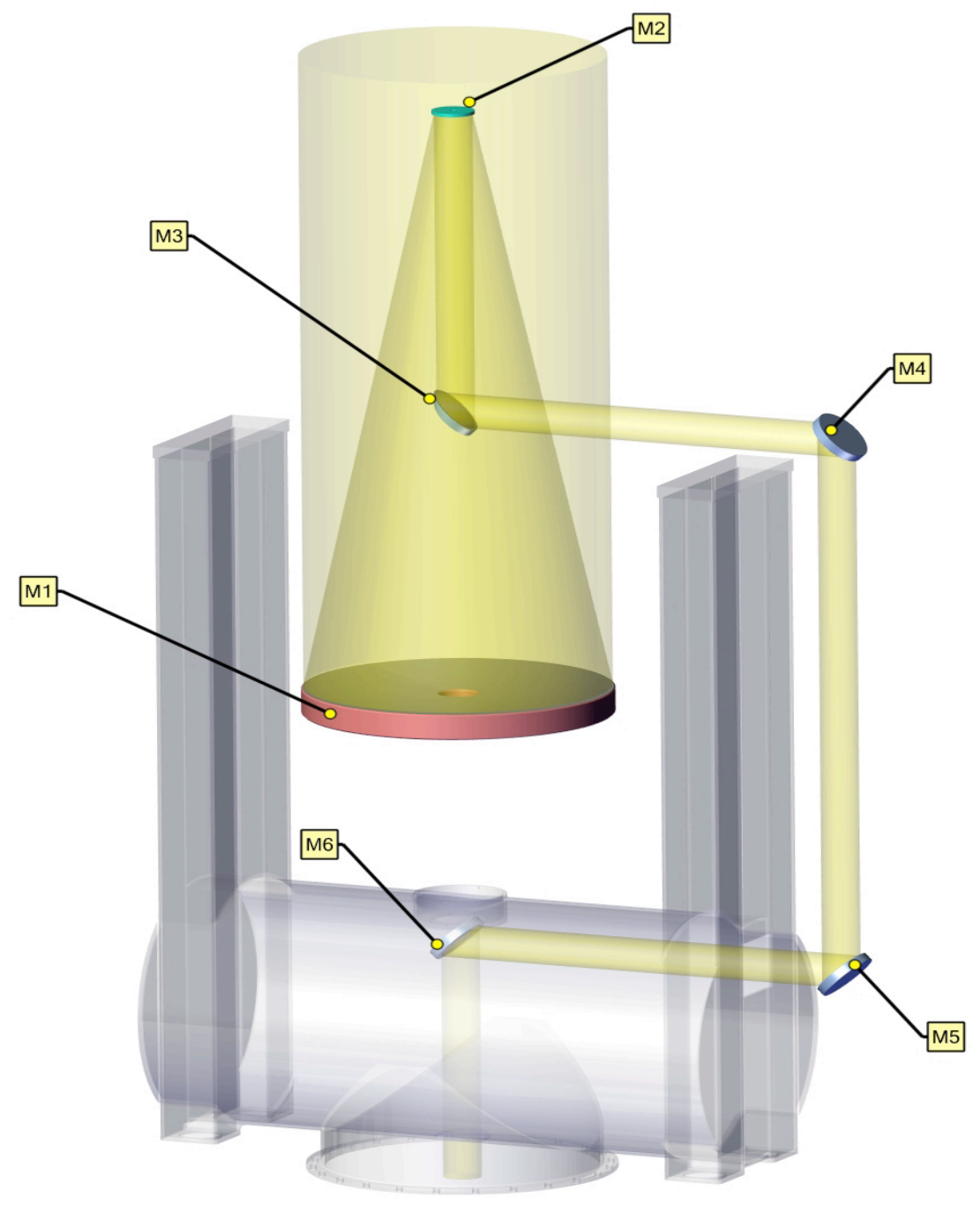

Figure 3. Beam path in the CHARA telescopes 


\subsection{Inserting the new system into the Coude path}

The optics of the six ALT-AZ Mersenne telescopes in the array consists of a $1 \mathrm{~m}$ concave paraboloidal primary mirror (M1) and a confocal $0.14 \mathrm{~m}$ convex paraboloidal secondary mirror (M2). The $0.125 \mathrm{~m}$ collimated output beam is guided along the elevation and azimuth axes of the telescope by flat mirrors M3, M4, M5 and M6. The central obscuration is $25 \%$. The output beam between M4 and M5 is easily accessible. This is the most convenient place for the new system to be installed.

Currently, the array operates with a PZT actuated M2 controlled by a tip/tilt detector in the laboratory ${ }^{2}$. On the one hand this arrangement is advantageous, because it can detect angular beam deviations in the whole optical train. On the other hand it suffers from low sensitivity due to losses on the numerous mirrors along the path. Placing a fast WFS for tip/tilt detection at the telescope will increase sensitivity, but necessitates a separate slow WFS system in the laboratory to measure non-common path errors between the telescope and the laboratory. The implementation of the slow WFS in the laboratory is not discussed in this paper.

\subsection{Optical layout and implementation of the fast wavefront sensing system}

The fast WFS system for TIP/TILT detection will be mounted between M4 and M5 on an optical breadboard. (Figure 4) The layout is fairly simple, inexpensive, and easy to align.

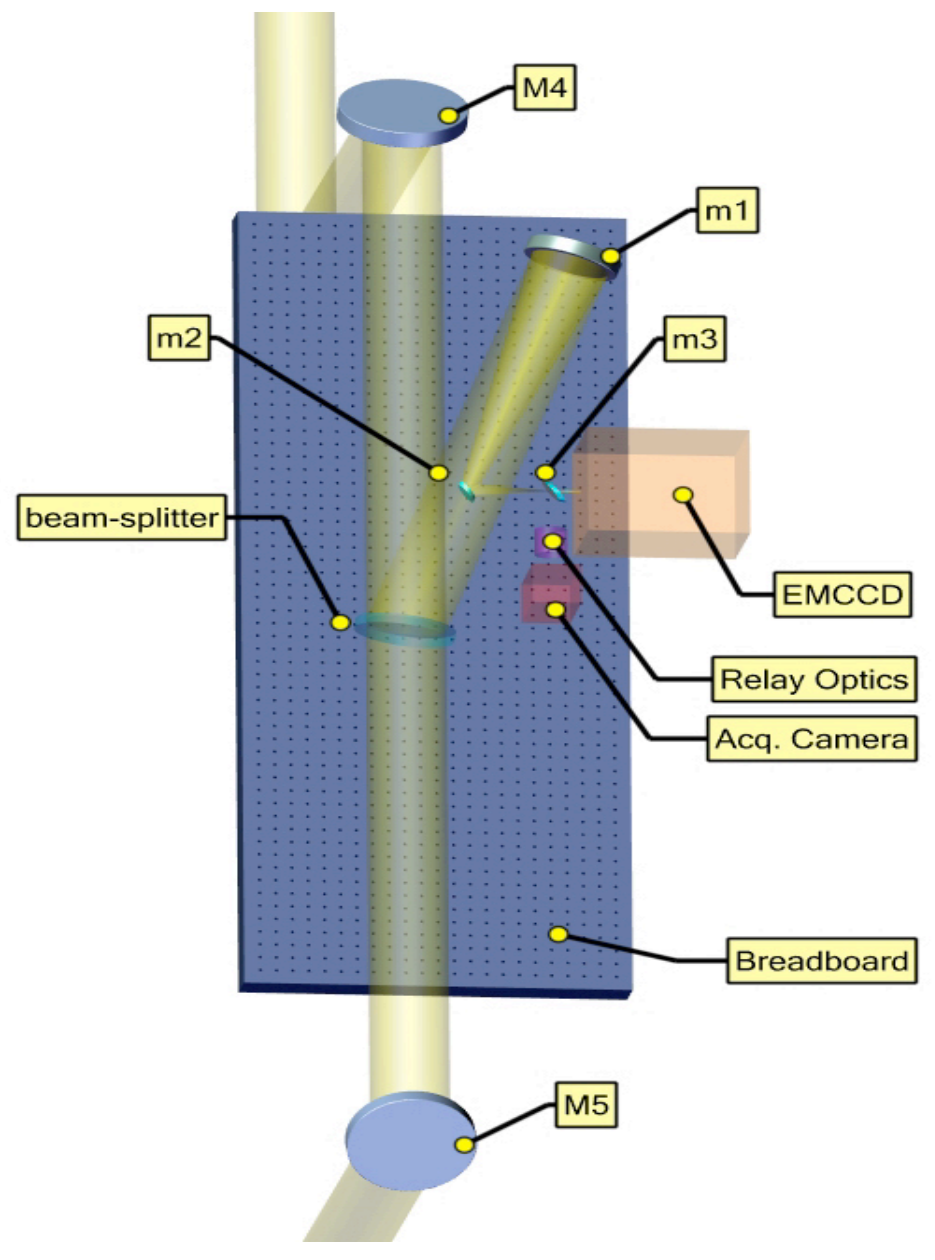

Figure 4. The new WFS and tip/tilt detection system installed on an optical breadboard on the telescope fork between M4 and M5 fold mirrors 
The first optical element in the new system is a beam-splitter. Since numerous beam combiners operating in different wavelength regimes are available at CHARA, there is a need to split the light in different ways between acquisition plus wavefront sensing and interferometry depending upon the beam combiner used. The current design can accommodate up to three beam-splitters on a remotely operated carousel. The detector is an EMCCD, thus one of the beam-splitters will be a dichroic mirror, which reflects most photons below 1 um toward the detector. The IR photons will be transmitted through the system for interferometry. The visible beam-combiners might use a gray split or a different dichroic split. The angle of incidence is 10 degrees on the beam-splitters. We also plan to use the other side of this dichroic as a means of injecting a laser beacan from the telescope back into the laboratory.

Light reflected by the dichroic is focused by a commercial 6 inch F/4 paraboloidal mirror (m1). Before the focus, a small elliptical flat mirror $(\mathrm{m} 2)$ intercepts the beam to place the focus in a more convenient place. The mirror is small enough to be hidden in the shadow cast by M3. The focus of the paraboloidal mirror falls in a small hole on another flat mirror (m3). Light from the science object goes through the hole undisturbed toward the WFS, while the rest of the field is reflected toward a relay optic followed by an image acquisition camera. A closeup of the optical path near the WFS can be seen in Figure 5. Light from the science object is collimated by a small lens, which also sets the diameter of the exit pupil. A $6 \times 6$ hexagonal lenslet array is placed in the exit pupil for focusing the starlight to 36 quad-pixels on the WFS camera. This camera will be used in photon-counting (high gain) mode for faint objects and in normal (low gain) mode for bright objects.

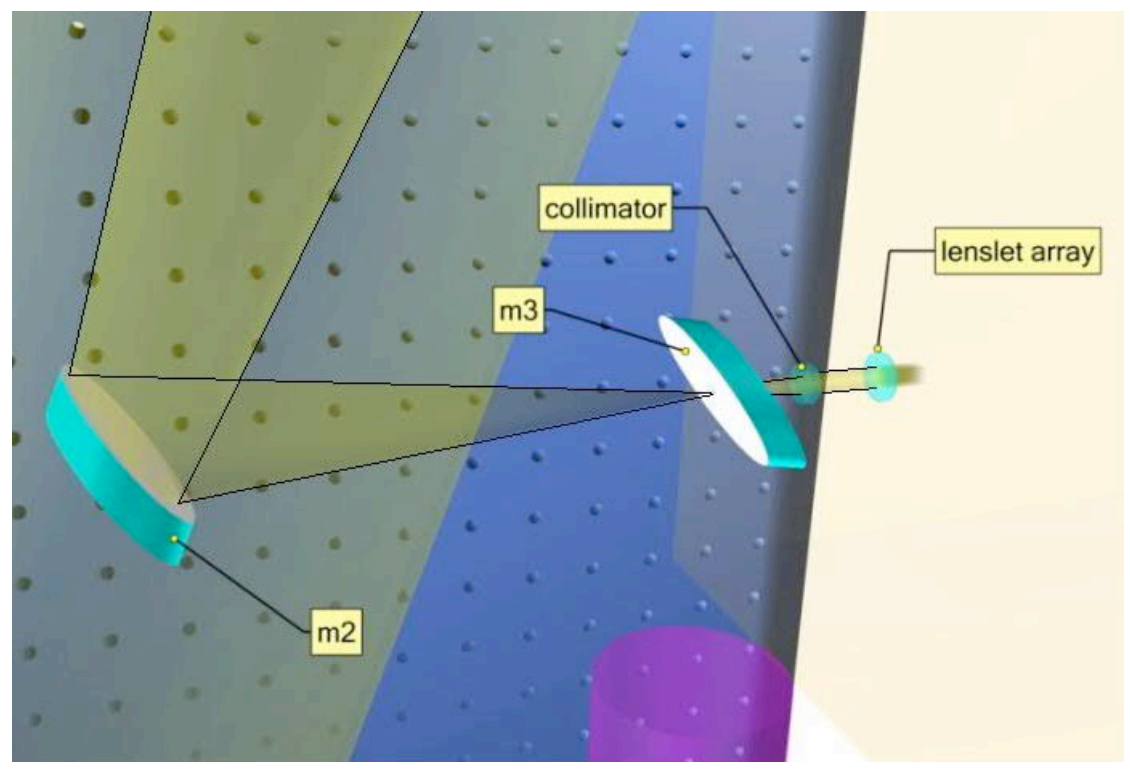

Figure 5. The light from the science object should go through the hole in the center of $\mathrm{m} 3$ mirror.

When the telescope is not yet pointing exactly to the science object, the light is reflected downward toward the acquisition camera, which is not shown in this closeup.

There are certain features of the old system which we have to keep in the new system. One of them is the use of a laser fiducial for co-aligning the telescope pointing axis and the rest of the optical system. The optical paths between the telescopes and beam-combiners are several hundred meters long and therefore the field of view in the laboratory is extremely narrow. Finding a star would be a daunting task without using an alignment laser for aligning the telescopes. A laser beam, nominally $\mathrm{D}=0.125 \mathrm{~m}$, can be sent out to each telescope from the laboratory. The same laser is used to align the beam-combiners in the laboratory. The laser beam is centered at the telescopes between M4 and M5. A small corner-cube in front of M2 intercepts the laser beam and sends a $25 \mathrm{~mm}$ beam back toward the image acquisition telescope. The acquisition camera picks up light from both the laser and from the target star so the observer sees a stationary "laser star" and the target star on the screen. The telescope is moved until the two images are coincident. Thus light from the target object follows the path of the laser and finds its way to the beam-combiner. The only difference between the new and old systems in this respect is that in the old system the corner-cube is centered in the beam and permanently mounted in front of M2, while it has to be off-center and movable in the new system otherwise the small 
flat mirrors in front of the paraboloidal mirror would block the laser beam from reaching the acquisition camera (Figure 6). When the object is acquired the corner cube is moved out of the beam. The moving mechanism can be simple, since the corner cube is not sensitive to alignment.

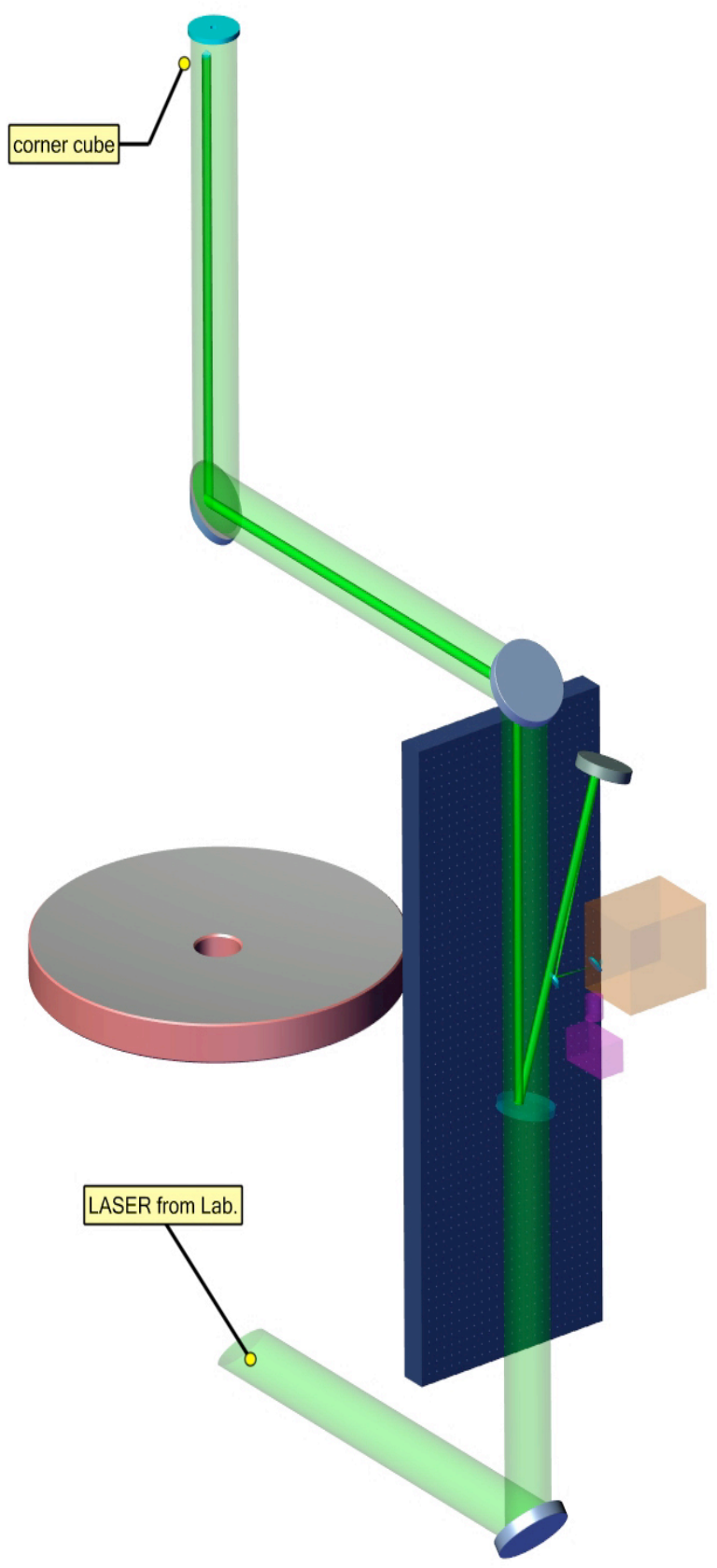

Figure 6. Modified path for the alignment laser

\subsection{Detector}

The camera was selected to meet the main science goal of observing fainter objects. At low-light levels, the read noise of the camera will dominate the signal to noise ratio and limit the sensitivity of the CHARA AO upgrade. The Electron Multiplication CCD (EMCCD) is the best detector identified so far in order to minimize this read noise. With Electron 
Multiplication on, the read noise of a typical EMCCD is less than 1 electron. Another criteria is the need for a fast frame rate. Typical seeing conditions at Mountain Wilson are $\mathrm{t}_{0} \sim 0.01 \mathrm{~s}$, which requires the frame rate to be faster than 100 frames per second. Because of the need for not only fast frame rates but also low latency, we chose to use the camera link data interface and a customized PCIe card to increase the data transferring speed and reduce the latency. The current design goal is for less than $50 \mu \mathrm{S}$ latency between the last of the pixel data being transferred via camera link interface and the data being available in memory via DMA transfer. The dichroic is specified to reflect most photons below 1 micron to the camera, therefore high Quantum Efficiency at these wavelengths is preferred. After an extensive search of available cameras, we have settled on the Andor iXon Ultra 897 with EX2 technology. We have one of these cameras currently under test in our laboratory.

\subsection{Future additions}

In Phase I, it is not essential to have a stimulus source available in the WFS system. However, it is essential in Phase II in order to calibrate the deformable mirror (DM). The DM most likely will replace M4. This way we can spare one extra reflection. The stimulus source will send a full size $(0.125 \mathrm{~m})$ beam downstream through the DM toward the wavefront sensor and the laboratory. It will likely be a fiber fed single aspheric lens mounted between M3 and the DM. The slow WFS and small deformable mirror at each of the six beams to measure and remove static aberrations and non-common path errors will be placed after the beam reducing telescopes in the laboratory where the beam diameter is reduced to 19 $\mathrm{mm}$.

\section{CURRENT STATUS}

The CHARA Array AO project is still in its early phase and we are largely still in the procurement stage of the long lead-time parts. The large optical substrates are now under construction and we have begun tests of the first CCD detector system. We expect work to begin on the first optical table later this year with first on-sky tests of the Phase I system to begin in early 2013.

\section{REFERENCES}

[1] ten Brummelaar, T.A., McAlister, H.A., Ridgway, S.T., Bagnuolo, W.G., Jr., Turner, N.H., Sturmann, L., Sturmann, J., Berger, D.H., Ogden, C.E., Cadman, R., Hartkopf, W.I., Hopper, C.H. \& Shure, M.A., "First Results from the CHARA Array. II. A Description of the Instrument", Ap.J., 628, 453-465 (2005).

[2] Sturmann, L., Sturmann, J., ten Brummelaar, T.A., \& McAlister, H.A., "Nine-Channel Tip/Tilt Detector at the CHARA Array,", Proc SPIE 6268, 62683T, (2006).

[3] ten Brummelaar, T.A., Ridgway, S.T. \& Bagnuolo W.G. Jr., "Strehl Ratio and Coherence Loss in Long Baseline Stellar Interferometry”, Opt. Lett., 20, 521 (1995)

[4] Lawson, P.R., Scott, T. R. \& Haniff, C. A., "Group-delay tracking and visibility fluctuations in long-baseline stellar interferometry", MNRAS, 304, 218, (1999)

[5] Dullemond, C.P. \& Monnier, J.D., "The Inner Regions of Protoplanetary Disks", ARA\&A, 48, 205, (2010)

[6] Millan-Gabet, R., Schloerb, F. P., Traub, W. A., Malbet, F., Berger, J. P. \& Bregman, J. D., "Sub-Astronomical Unit Structure of the Near-Infrared Emission from AB Aurigae", Ap.J., 513, L131, (1999)

[7] Millan-Gabet, R., Schloerb, F. P., \& Traub, W.A., "Spatially Resolved Circumstellar Structure of Herbig AE/BE Stars in the Near-Infrared", Ap.J., 546, 358, (2001)

[8] Monnier, J., \& Millan-Gabet, R., "On the interferometric sizes of young stellar objects”, Ap.J. ,579, 694-698 (2002).

[9] Natta, A., Prusti, T., Neri, R., Wooden, D., Grinin, V. P., \& Mannings, V., “A reconsideration of disk properties in Herbig Ae stars", A\&A, 371, 186-197, (2001)

[10] Dullemond, C.P., Dominik, C. \& Natta, A., Passive Irradiated Circumstellar Disks with an Inner Hole, Ap.J., 560, 957-969, (2001)

[11] Akeson, R. L., Boden, A. F., Monnier, J. D., Millan-Gabet, R., Beichman, C., Beletic, J., Calvet, N., Hartmann, L., Hillenbrand, L., Koresko, C., Sargent, A., Tannirkulam, A., "Keck Interferometer observations of Classical and Weak-lined T Tauri Stars", Ap.J., 635, 1183-1181, (2005)

[12] Eisner, J., Hillenbrand, White, Bloom, Akeson, \& Blake, "Near-infrared interferometric, spectroscopic, and photometric monitoring of T Tauri inner disks", Ap.J., 669, 1072, (2007)

[13] Pinte, C., Menard, F., Berger, J.Pl, Benisty, M., \& Malbet, F., "The inner radius of T Tauri Disks estimated 
from Near-IR Interferometry: The Importance of Scattered Light”, Ap.J.Let. , 673, L63-66, (2008)

[14] Millan-Gabet, R., Malbet, F., Akeson, R., Leinert, C., Monnier, J., Waters, R., "The circumstellar environments of young stars at AU scales", Proc. Protostars and Planets V, 951, 539-554, (2007)

[15] Kenyon, S. \& Hartmann, L. "Pre-Main-Sequence Evolution in the Taurus-Auriga Molecular Cloud", Ap.J.Sup., 101, 117, (1995).

[16] Swain, M., Vasisht, G., Akeson, R., Monnier, J., Millan-Gabet, R., Serabyn, E., Creech-Eakman, M., van Belle, G., Beletic, J., Beichman, C., Boden, A., Booth, A., Colavita, M., Gathright, J., Hrynevych, M., Koresko, C., Le Mignant, D., Ligon, R., Mennesson, B., Neyman, C., Sargent, A., Shao, M., Thompson, R., Unwin, S. and

Wizinowich, P., "Interferometer observations of subparsec-scale infrared emission in the nucleus of NGC 4151.", Ap.J. 596, L163-166, (2003).

[17] Kishimoto, M., Honig, S., Tristram, K., \& Weigelt, G., "Possible evidence for a common radial structure in nearby AGN tori”, A\&A, 493, 57-60, (2009)

[18] Nenkova, M., Ivezic, Z., \& Elitzur, M., "Dust emission from active galactic nuclei", Ap.J.Let., 570, L9-12, (2002)

[19] Keller, Christoph U.; Plymate, Claude; Ammons, S. M., "Low-cost solar adaptive optics in the infrared", Proc SPIE 4853, 351 (2003) 